\title{
Lattice-QCD determination of the hyperon axial couplings in the continuum limit
}

\author{
Aditya Savanur ${ }^{1}$ and Huey-Wen Lin ${ }^{1,2, *}$ \\ ${ }^{1}$ Department of Physics and Astronomy, Michigan State University, East Lansing, Michigan 48824, USA \\ ${ }^{2}$ Department of Computational Mathematics, Michigan State University, \\ East Lansing, Michigan 48824, USA
}

(Received 21 January 2019; accepted 22 June 2020; published 2 July 2020)

\begin{abstract}
We present the first chiral-continuum-finite-volume extrapolation of the hyperon octet axial couplings $\left(g_{\Sigma \Sigma}\right.$ and $\left.g_{\Xi \Xi}\right)$ from $N_{f}=2+1+1$ lattice QCD. These couplings are important parameters in the low-energy effective field theory description of the octet baryons and are fundamental to the nonleptonic decays of hyperons and to hyperon-hyperon and hyperon-nucleon scattering with applications to neutron stars. We use clover lattice fermion action for the valence quarks with sea quarks coming from configurations of $N_{f}=2+1+1$ highly improved staggered quarks generated by MILC Collaboration. Our work includes the first calculation of $g_{\Sigma \Sigma}$ and $g_{\Xi \Xi}$ directly at the physical pion mass on the lattice, and a full account of systematic uncertainty, including excited-state contamination, finite-volume effects, and continuum extrapolation, all addressed for the first time. We find the ratio of the continuum-limit hyperon coupling constants with respect to the nucleon axial charges to be $g_{\Sigma \Sigma} / g_{A}=0.351(6)(2)$ and $g_{\Xi \Xi} / g_{A}=-0.213(5)(1)$, where the first error includes the statistical and extrapolation systematic uncertainties, and the second error corresponds to systematics associated with varying lattice parameters. The $\mathrm{SU}(3)$ symmetry breaking derived from these axial charges is $9 \%$, which is about a factor of 2 smaller than earlier lattice estimates.
\end{abstract}

DOI: 10.1103/PhysRevD.102.014501

\section{INTRODUCTION}

The octet-baryon axial couplings $\left(g_{\Sigma \Sigma}, g_{\Xi \Xi}\right.$, and $\left.g_{A}\right)$ are important quantities for studying hadron structure in QCD. Specifically, the hyperon couplings are important in the effective field theory of octet baryons [1], because they enter the expansions of all quantities in chiral perturbation theory. In addition, the coupling constants appear in the calculations of hyperon nonleptonic decays [2] and of hyperon-hyperon and hyperon-nucleon scattering matrix elements [3]. They are also useful for calculating equations of state and other properties of nuclear matter in neutron stars $[4,5]$. Studying these couplings allows us to explore the extent of the symmetry breaking of SU(3) flavor. SU(3) symmetry has been widely studied [1,6] in the hyperon hadronic matrix elements, and this symmetry is used in many applications where strange-quark data are limited. For example, the global analysis of the polarized parton distribution function (PDF) has commonly used this assumption for extracting individual quark flavor PDFs

\footnotetext{
*hwlin@pa.msu.edu
}

Published by the American Physical Society under the terms of the Creative Commons Attribution 4.0 International license. Further distribution of this work must maintain attribution to the author(s) and the published article's title, journal citation, and DOI. Funded by SCOAP ${ }^{3}$.
[7]; knowing to what extent this symmetry holds will help us quantify the systematic uncertainty introduced by the use of this assumption in the polarized PDF [7]. However, experimentally it is much harder to determine the hyperon couplings than those in the nucleon case, since the hyperons weak decay in nature quickly. Lattice-QCD (LQCD) calculations can provide more stringent direct and reliable calculations of these couplings.

Lattice QCD is an ideal theoretical tool to study the parton structure of hadrons, starting from quark and gluon degrees of freedom (d.o.f). Progress has long been limited by computational resources, but recent advances in both algorithms and a worldwide investment in pursuing exascale computing has led to exciting progress in LQCD calculations. Take the nucleon tensor charge for example. Experimentally, one gets the tensor charges by taking the zeroth moment of the transversity distribution; however, the transversity distribution is poorly known and such a determination is not very accurate. On the lattice side, there are a number of calculations of $g_{T}$ [8-16]; some of them are done with more than one ensemble at physical pion mass with high-statistics calculations (about 100k measurements) and some with multiple lattice spacings and volumes to control lattice artifacts. Such programs would have been impossible 5 years ago. As a result, the latticeQCD tensor-charge calculation has the most precise determination of this quantity, which can then be used to 
constrain the transversity distribution and make predictions for upcoming experiments [17]. The hyperon couplings are also not precisely known from experiments, and we hope a better determination of these couplings will lead to advancements in multiple subfields.

In this work, we use the following definitions for the axial couplings:

$$
\begin{aligned}
g_{A} & =Z_{A}\left\langle N\left|A_{\mu}\right| N\right\rangle^{\text {lat },} \\
g_{\Sigma \Sigma} & =Z_{A}\left\langle\Sigma\left|A_{\mu}\right| \Sigma\right\rangle^{\text {lat }} / 2, \\
g_{\Xi \Xi} & =Z_{A}\left\langle\Xi\left|A_{\mu}\right| \Xi\right\rangle^{\text {lat },}
\end{aligned}
$$

where $Z_{A}$ is the renormalization constant for the axial current. The factor of 2 in $g_{\Sigma \Sigma}$ comes from a ClebschGordan coefficient so that $g_{\Sigma \Sigma}=F$ in the SU(3) limit. The octet axial structure can be obtained through

$$
\left\langle B\left|A_{\mu}(q)\right| B\right\rangle=u_{B}\left(p^{\prime}\right)\left[\gamma_{\mu} \gamma_{5} G_{A}\left(q^{2}\right)+\gamma_{5} q_{\mu} \frac{G_{P}\left(q^{2}\right)}{2 M_{B}}\right] u_{B}(p),
$$

where $B$ is an octet baryon $(N, \Sigma, \Xi), u_{B}$ is the Dirac spinor, $G_{A}$ is the axial form factor, $G_{P}$ is the induced pseudoscalar form factor, and $q=p^{\prime}-p$ is the transfer momentum. In the $q^{2}=0$ limit, we obtain the octet coupling constants that come from $G_{A}\left(q^{2}=0\right)$.

There have been many lattice-QCD calculations of nucleon axial charges in the past few decades (see Ref. [18] for a detailed review) but only a few calculations of the hyperon couplings. The first such calculation was performed in 2007 with a single lattice spacing and lightest pion mass near $350 \mathrm{MeV}$ [19] using 2+1-flavor lattices; they got $g_{\Sigma \Sigma}=$ $0.450(21)_{\text {stat }}(27)_{\text {sys }}$ and $g_{\Xi \Xi}=-0.277(15)_{\text {stat }}(19)_{\text {sys }}$. A follow-up study by a Japanese group used $N_{f}=2$ lattices [20], producing results consistent with the heavier pion masses of Ref. [19]. ETMC [21] used $N_{f}=2+1+1$ lattices with lowest pion mass $213 \mathrm{MeV}$ and two lattice spacings, 0.082 and $0.065 \mathrm{fm}$; they obtained $g_{\Sigma \Sigma}=0.381(11)$ and $g_{\Xi \Xi \Xi}=$ $-0.248(9)$ (statistical errors only) after extrapolating to the physical pion mass. In this work, we not only present the first calculation of these quantities at physical pion mass, but also study the finite-volume effects and lattice discretization systematics, and report the first continuum-limit results for the hyperon axial couplings.

\section{LATTICE-QCD CALCULATION SETUP}

In this work, we use clover lattice fermion action for the valence quarks on top of $2+1+1$ flavors $^{1}$ of hypercubic

\footnotetext{
${ }^{1}$ The notation " $2+1+1$ " indicates that the $\mathrm{QCD}$ vacuum contains dynamical quark degrees of freedom with degenerate up and down quarks plus strange and charm quarks at their physical masses.
}

(HYP)-smeared [22] highly improved staggered quarks (HISQ) [23,24] in configurations generated by MILC Collaboration. The quark masses for the clover fermions have been tuned to reproduce the lightest sea staggered pseudoscalar meson masses for the light and strange quarks, and the clover parameters are set to the tree-level tadpole-improved values; a similar setup was used by PNDME Collaboration in many studies of nucleon structure [8]. We use three lattice spacings $a, 0.06,0.09$, and $0.12 \mathrm{fm}$ and pion masses $M_{\pi}$ ranging from near physical pion mass $(135 \mathrm{MeV})$ to around $310 \mathrm{MeV}$. We also perform a volume-dependence study at $a \approx 0.12 \mathrm{fm}$ and $M_{\pi} \approx$ $220 \mathrm{MeV}$ where $M_{\pi} L$ ranges from 3.3 to 5.5. A summary of the ensemble parameters used in our calculations can be found in Table I.

Any mixed-action approach results in a nonunitary lattice-QCD formulation with the possibility of exceptional configurations. Signatures of such configurations, which manifest at sufficiently small quark mass, include correlation functions with anomalously large values that bias the ensemble average and failure of the clover Dirac matrix solver to converge due to poor condition number. The two signatures have been observed at $a \approx 0.15$ and $0.12 \mathrm{fm}$ at $M_{\pi} \approx 135 \mathrm{MeV}$, and these ensembles are excluded from use in mixed-action calculations. The other ensembles are carefully checked for the relevant signatures, and exceptional configurations are absent for the $M_{\pi} \in\{220,310\} \mathrm{MeV}$ MILC ensembles [24] at $0.12 \mathrm{fm}$ and finer lattice spacings, as well as for 0.09 and $0.06 \mathrm{fm}$ near the physical pion mass. There are no issues that we have observed for any observable on the ensembles used in this calculation.

Another complication associated with mixed action may occur in the continuum extrapolation, and a mixed-action partially quenched formulation would be needed to remove such artifacts. For the nucleon axial charges [25,26], the partially quenched terms appear with terms proportional to the difference between the squares of the valence and sea pion masses, $\left(M_{\pi}^{\mathrm{val}}\right)^{2}-\left(M_{\pi}^{\text {sea }}\right)^{2}$. Since we tuned the valence pion mass to be the same as the sea one when setting the parameters for the valence fermion actions, the remaining partially quenched effect comes at $O\left(a^{2}\right)$. We will use our data to estimate the systematics by varying continuum

TABLE I. Ensemble information and parameters used in this calculation.

\begin{tabular}{lcccrc}
\hline \hline Ensemble ID & $L_{s}^{3} \times L_{t}$ & $M_{\pi}^{\text {val }} L_{s}$ & $t_{\text {sep }} / a$ & $N_{\text {conf }}$ & $N_{\text {meas }}$ \\
\hline a12m310 & $24^{3} \times 64$ & 4.5 & $\{8,9,10,11,12\}$ & 1013 & 4052 \\
a12m220S & $24^{3} \times 64$ & 3.3 & $\{8,10,12\}$ & 1000 & 6000 \\
a12m220 & $32^{3} \times 64$ & 4.4 & $\{8,10,12\}$ & 958 & 3832 \\
a12m220L & $40^{3} \times 64$ & 5.5 & $\{10\}$ & 1010 & 4040 \\
a09m310 & $32^{3} \times 64$ & 4.5 & $\{10,12,14\}$ & 775 & 3100 \\
a09m220 & $48^{3} \times 64$ & 4.8 & $\{10,12,14\}$ & 890 & 3560 \\
a09m130 & $64^{3} \times 64$ & 3.9 & $\{10,12\}$ & 1058 & 4232 \\
a06m310 & $48^{3} \times 64$ & 4.5 & $\{13,16,20\}$ & 480 & 1920 \\
\hline \hline
\end{tabular}


extrapolation by replacing $M_{\pi}^{\text {val }}$ by $M_{\pi}^{\text {sea }}$ and also include $O\left(a^{2}\right)$ to estimate the size of such contribution. Unfortunately, there is no similar mixed-action chiral form derived for hyperon axial couplings; we assume the effects would be similar to nucleon case, arising from terms proportional to $\left(M_{K}^{\text {val }}\right)^{2}-\left(M_{K}^{\text {sea }}\right)^{2}$; we plan to use similar strategies in the nucleon case to estimate such systematics.

Despite these caveats, mixed-action calculations of nucleon properties have shown consistent results with unitary-action calculations with similar lattice parameters (lattice spacing, pion mass, etc.). In the case of the nucleon axial charge, for example, the mixed-action results by PNDME $[8,10,11]$ are consistent with those from cloveron-clover calculations $[27,28]$ at similar pion masses. The most recent review of nucleon charges across all lattice calculations shows consistent determinations between mixed action and unitary fermion actions [29]. This suggests there is unlikely to be any significant mixedaction artifacts (outside statistical errors) in the chiral/ continuum extrapolation of the hyperon couplings.

We choose the following baryonic interpolating operator with the quantum numbers of a spin- $1 / 2$ baryon:

$$
\chi^{N}(x)=\epsilon^{a b c}\left[q_{1}^{a \top}(x) C \gamma_{5} q_{2}^{b}(x)\right] q_{1}^{c}(x),
$$

where $C$ is the charge-conjugation matrix $i \gamma_{4} \gamma_{2},\{a, b, c\}$ are color indices, $\epsilon$ is the antisymmetric tensor, and $q_{1}$ and $q_{2}$ are one of the three quarks $\{u, d, s\}$. For example, in the case of the proton $/ \Sigma / \Xi$, we have $q_{1}=u / u / s$ and $q_{2}=d / s / d$.

To extract the octet axial couplings, we simultaneously fit the octet two-point $\left(C_{2 \mathrm{pt}}\right)$ and three-point $\left(C_{3 \mathrm{pt}}\right)$ correlators, including the first excited state, to the form

$$
\begin{aligned}
C^{2 \mathrm{pt}}(t) & =\left|\mathcal{A}_{0}\right|^{2} e^{-M_{0} t}+\left|\mathcal{A}_{1}\right|^{2} e^{-M_{1} t}, \\
C_{\Gamma}^{3 \mathrm{pt}}\left(t, t_{\mathrm{sep}}\right)= & \left|\mathcal{A}_{0}\right|^{2}\left\langle 0\left|\mathcal{O}_{\Gamma}\right| 0\right\rangle e^{-M_{0} t_{\text {sep }}} \\
& +\left|\mathcal{A}_{1}\right|^{2}\left\langle 1\left|\mathcal{O}_{\Gamma}\right| 1\right\rangle e^{-M_{1} t_{\text {sep }}} \\
& +\mathcal{A}_{1} \mathcal{A}_{0}^{*}\left\langle 1\left|\mathcal{O}_{\Gamma}\right| 0\right\rangle e^{-M_{1}\left(t_{\mathrm{sep}}-t\right)} e^{-M_{0} t} \\
& +\mathcal{A}_{0} \mathcal{A}_{1}^{*}\left\langle 0\left|\mathcal{O}_{\Gamma}\right| 1\right\rangle e^{-M_{0}\left(t_{\text {sep }}-t\right)} e^{-M_{1} t}
\end{aligned}
$$

where $A_{0}$ and $A_{1}$ are overlap amplitudes for the ground and excited states, $t_{\text {sep }}$ is the source-sink separation, and $M_{0}$ and $M_{1}$ are the masses for ground and excited states of the corresponding octet baryons. For $q^{2}=0$, the third and fourth terms are related, so Eq. (5) can be combined into a three-term fit. Note that the unwanted matrix element $\left\langle 1\left|\mathcal{O}_{\Gamma}\right| 1\right\rangle$ has the same time dependence as the wanted ground-state matrix element $\left\langle 0\left|\mathcal{O}_{\Gamma}\right| 0\right\rangle$, so this excited-state contamination can only be reliably extracted when there are multiple $t_{\text {sep }}$ in the data. We fit the matrix elements from all ensembles up to $\left\langle 1\left|\mathcal{O}_{\Gamma}\right| 1\right\rangle$ with the exception of the a $12 \mathrm{~m} 220 \mathrm{~L}$ ensemble for which only one source-sink separation was taken; only two-term fits (up to $\left\langle 1\left|\mathcal{O}_{\Gamma}\right| 0\right\rangle$ ) can be used to extract the bare matrix elements. The $\chi^{2} /$ d.o.f of these fits range from 0.4 to 1.2. Figure 1 shows the ratio plots $C_{3 \mathrm{pt}} / C_{2 \mathrm{pt}}\left(t_{\mathrm{sep}}\right)$ of a few selected octet couplings and the extracted ground-state matrix elements.

We can validate our omission of the excited-excited term from the a12m220L ensemble fits by considering the results of using the same fits on ensembles for which additional separations are available. We have three sourcesink separations for a $12 \mathrm{~m} 220 \mathrm{~S}$ and $\mathrm{a} 12 \mathrm{~m} 220$ ensembles, in which we found our extracted ground-state nucleon $g_{A}$ obtained from using only $t_{\text {sep }}=10$ are consistent with those using all three $t_{\text {sep }}$. Thus, there is no sign of excitedstate contamination from the inextricable $\left\langle 1\left|\mathcal{O}_{\Gamma}\right| 1\right\rangle$ term at the chosen source-sink separation. Another check comes from an independent study by PNDME Collaboration [8] using the same quark smearing parameters and multiple source-sink separations (including the ones used in this work). Figure 8 in Ref. [8] shows a consistent extraction between ground-state nucleon axial charge from two-state analysis of the three-point data only at $t_{\text {sep }}=0.96 \mathrm{fm}$ without $\left\langle 1\left|\mathcal{O}_{\Gamma}\right| 1\right\rangle$ compared to a simultaneous fit to all

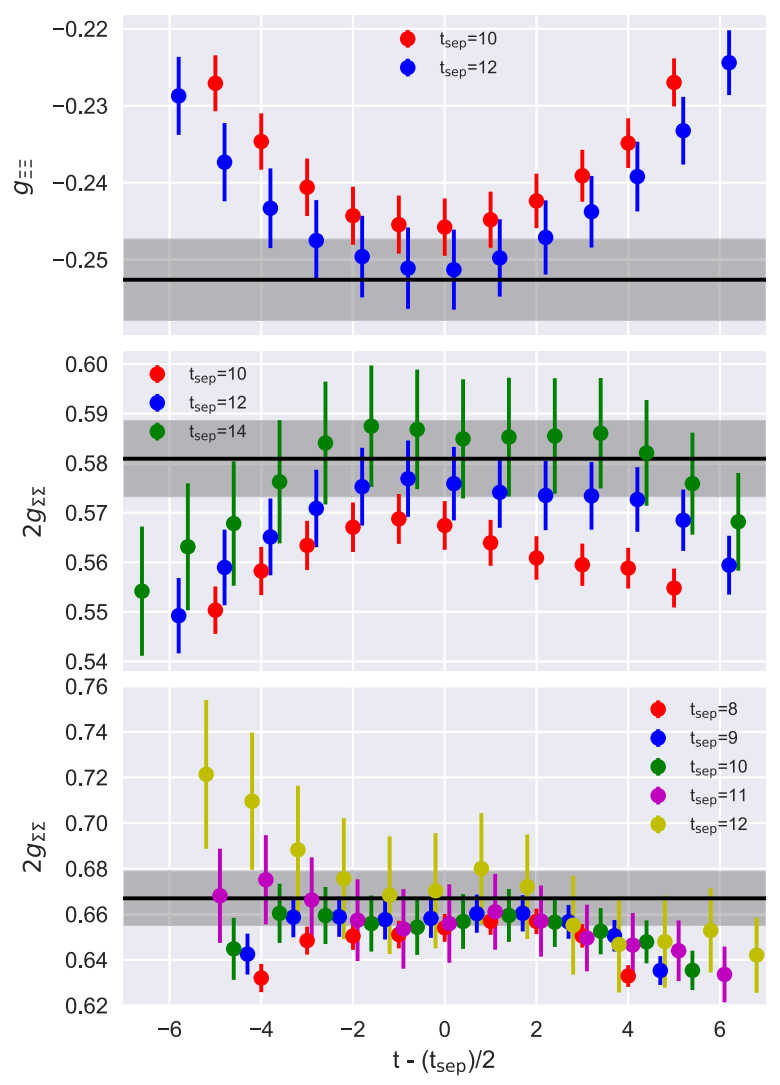

FIG. 1. Example ratio plots of $C_{3 \mathrm{pt}} / C_{2 \mathrm{pt}}\left(t_{\mathrm{sep}}\right)$ for $g_{\Xi \Xi}$ (top, a09m130 ensemble) and $g_{\Sigma \Sigma}$ (middle and bottom, a09m220 and a12m310, respectively). The extracted ground-state hyperon matrix elements from two-state fit using all the $t_{\text {sep }}$ are marked by the black line and gray band. 
TABLE II. Our renormalized isovector nucleon axial charge $g_{A}$ obtained from the $2+1+1$-flavor HISQ ensembles, compared with other calculations done on the same ensembles with clover valence fermions by PNDME [8] and with domain-wall fermions by the CalLat Collaboration [30]. Our results are consistent within $1 \sigma$ with both collaborations who use much larger statistics, their goal being to make a precision calculation to compare with the subpercent experimental measurements of the nucleon axial charge.

\begin{tabular}{cccc}
\hline \hline & This work & PNDME18 [8] & CalLat18 [30] \\
\hline a12m310 & $1.239(36)$ & $1.251(19)$ & $1.214(13)$ \\
a12m220S & $1.273(79)$ & $1.224(44)$ & $1.272(28)$ \\
a12m220 & $1.242(45)$ & $1.234(25)$ & $1.259(15)$ \\
a12m220L & $1.279(40)$ & $1.262(17)$ & $1.252(21)$ \\
a09m310 & $1.240(47)$ & $1.235(15)$ & $1.236(11)$ \\
a09m220 & $1.249(50)$ & $1.260(19)$ & $1.253(09)$ \\
a09m130 & $1.235(62)$ & $1.249(21)$ & $N / A$ \\
a06m310 & $1.192(53)$ & $1.205(24)$ & $N / A$ \\
\hline \hline
\end{tabular}

$t_{\text {sep }} \in\{0.96,1.08,1.20,1.44,1.56\} \mathrm{fm}$ including $\left\langle 1\left|\mathcal{O}_{\Gamma}\right| 1\right\rangle$. The latter analysis with more input data merely results in a more precise extraction of the nucleon axial charge.

\section{NUCLEON ISOVECTOR AXIAL CHARGES ON HISQ LATTICES}

Our nucleon axial coupling results are consistent with those obtained by PNDME Collaboration [8]. In Table II, we compare the nucleon axial couplings obtained from our calculation with PNDME Collaboration [8] and CalLat [30], who also used MILC HISQ ensembles. We used two definitions to determine the renormalized nucleon axial couplings, a similar procedure as used by PNDME Collaboration [10]. We calculated both $g_{A}^{\text {ren }}=Z_{A} g_{A}^{\text {bare }}$ and $g_{A}^{\text {ren }}=Z_{A} / Z_{V} g_{A}^{\text {bare }} / g_{V}^{\text {bare }}$, where $Z_{A}$ and $Z_{V}$ are renormalization constants taken from Ref. [10] and $g_{V}$ is the vector charge. The difference between these two determinations is folded into the final error shown in Table II. Note that PNDME Collaboration used the all-mode averaging technique to achieve greater precision using $O(10-100 \mathrm{k})$ measurements, which is important in pursuing precision determination of the nucleon charges; as a result, PNDME has a more precise nucleon axial coupling determination than we do. However, no calculation of the hyperon couplings or SU(3) symmetry on these lattices has been done before. Using the same continuum-limit extrapolation as Ref. [17], we obtained nucleon axial charge coupling $g_{A}=1.22(6)$. Since our focus of this work in on hyperons, we refer interested readers to Ref. [8] (and references therein) for more detailed discussion of the nucleon axial charge.

\section{RESULTS AND DISCUSSION}

In this work, we choose to use ratios of hyperon to nucleon axial couplings for continuum extrapolation. Such a ratio choice has the following advantages: As discussed in the PNDME works $[8,10,11]$, there is a sizable uncertainty introduced by the nonperturbative renormalization of the axial-current operator in RI/MOM scheme. Table IX of Ref. [11] shows renormalization constants obtained in $\overline{\mathrm{MS}}$ scheme with two different choices of parameters in the RIsMOM scheme. Within each method, the errors for $Z_{A}$ are subpercent; however, when comparing the spread among different choices, we find the renormalization factors carry about 5\% systematic. This is responsible for the larger errors quoted in PNDME's final nucleon axial charge over that from CalLat [30], who used chiral fermions to avoid the axial-current renormalization procedure. In addition to avoiding the additional uncertainty due to nonperturbative renormalization described above, the signal-to-noise of the ratios is significantly improved due to the correlations in the data, since they are taken using the same QCD configurations. Furthermore, we expect some lattice artifacts to be canceled or reduced in the ratio combinations. Figures 2 and 3 summarize all our data as functions of $M_{\pi}^{2}$, lattice spacing $a$, and the dimensionless volume-dependent parameter $M_{\pi} L$. As shown in Fig. 2, we found the results at $310-\mathrm{MeV}$ pion mass show some lattice-spacing dependence but are consistent within the statistical error. The ratio of the a09m220 ensemble is consistent with those obtained from a12m220, which has similar $M_{\pi} L$, as well as
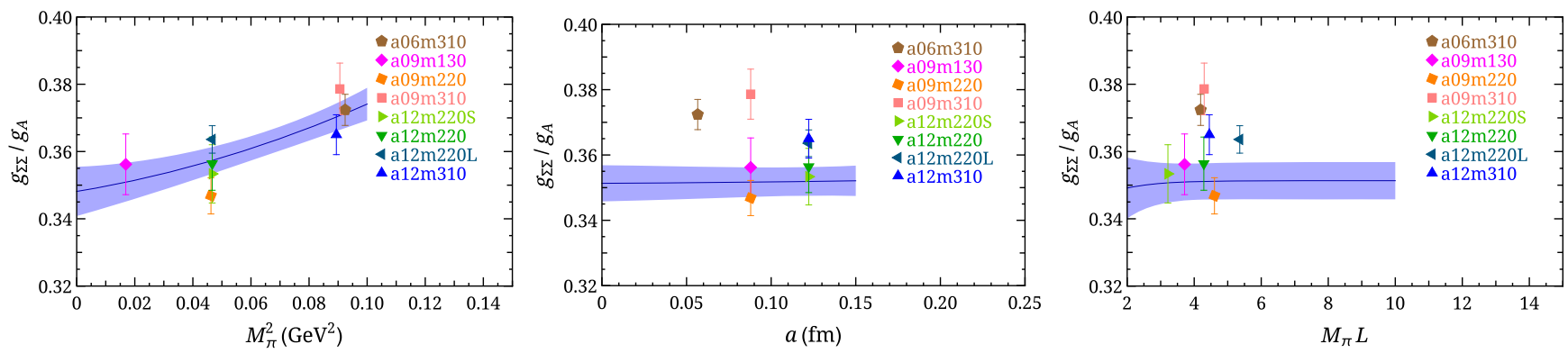

FIG. 2. Ratios of the $\Sigma$ axial coupling to the nucleon axial coupling as functions of $M_{\pi}^{2}$, lattice spacing $a$, and volume dependence in dimensionless $M_{\pi} L$. In each plot, the blue line and band show the fit using 12 simultaneous mass-lattice-spacing-volume extrapolations combining using AIC versus $M_{\pi}^{2}$ (left), $a$ (right), $M_{\pi} L$ (right), with the data projected in the other variables to $a=0, M_{\pi} L \rightarrow \infty$ (left), $M_{\pi}=135 \mathrm{MeV}, M_{\pi} L \rightarrow \infty$ (middle), and $M_{\pi}=135 \mathrm{MeV}, a=0$ (right). 

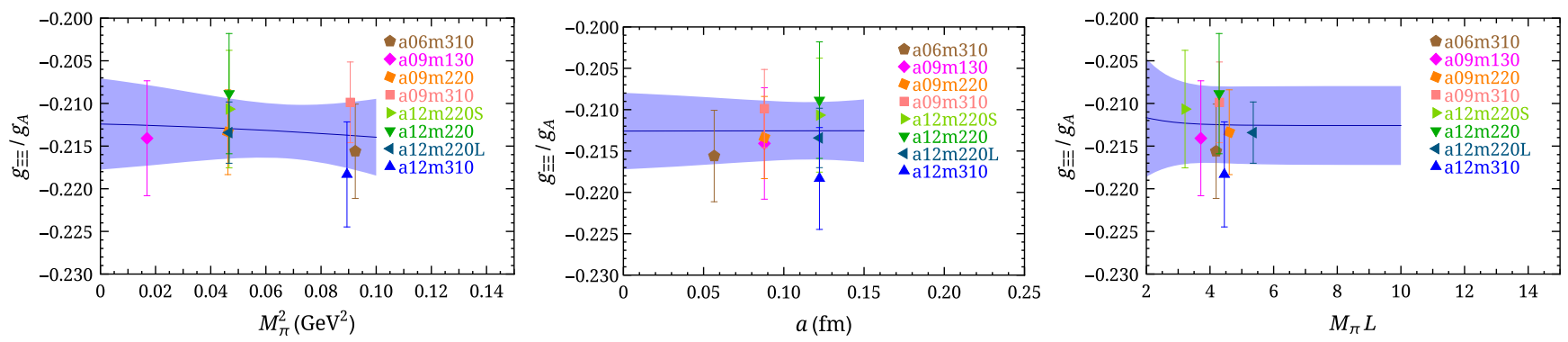

FIG. 3. Ratios of the $\Xi$ axial coupling to the nucleon axial coupling as functions of $M_{\pi}^{2}$, lattice spacing $a$, and volume dependence in dimensionless $M_{\pi} L$. In each plot, the blue line and band show the fit using 12 simultaneous mass-lattice-spacing-volume extrapolations combining using AIC versus $M_{\pi}^{2}$ (left), $a$ (right), $M_{\pi} L$ (right), with the data projected in the other variables to $a=0, M_{\pi} L \rightarrow \infty$ (left), $M_{\pi}=135 \mathrm{MeV}, M_{\pi} L \rightarrow \infty$ (middle), and $M_{\pi}=135 \mathrm{MeV}, a=0$ (right).

a12m220S, which has smaller volume; this indicates small lattice-spacing dependence. There is, however, $2.5 \sigma$ tension with the larger-volume data from a12m $220 \mathrm{~L}$. Given the lack of a consistent trend among the ensembles, the low value of a09m220 is likely caused by statistical fluctuation. As for the volume dependence, we do not see significant lattice-spacing nor volume dependence in these ratios. In the $\Xi$ case, we see very little dependence on pion mass, lattice spacing or volume, as shown in Fig. 3.

To extrapolate to the physical limit, we use the following general form:

$$
\begin{aligned}
\frac{g_{\Sigma \Sigma, \Xi \Xi \Xi}}{g_{A}}\left(M_{\pi}, a, L\right)= & c_{0}+c_{M} f_{M}\left(M_{\pi}^{2}\right)+c_{a} f_{a}(a) \\
& +c_{V} f_{V}\left(M_{\pi} L\right),
\end{aligned}
$$

which accounts for the lattice spacing $a$, pion mass $M_{\pi}$, and volume dependence $M_{\pi} L$. For each hyperon coupling ratio, $g_{\Sigma \Sigma} / g_{A}$ and $g_{\Xi \Xi} / g_{A}$, we vary the pion mass, volume, and lattice-spacing dependence among $\left\{M_{\pi}^{2}, M_{\pi}^{4}\right\},\left\{0, e^{-M_{\pi} L}\right\}$ and $\left\{0, a, a^{2}\right\}$, respectively. This yields 12 possible extrapolation forms, each of which has $\chi^{2} /$ d.o.f $<1$.

Rather than choosing a single fit to best represent the extrapolation from our data, we can use the Akaike information criterion (AIC) to combine the results of all these fits according to how well they fit the data. This method assigns a weight to each fit depending on the sum of the squared fit residuals $\chi^{2}$, and its number of degrees of freedom $k$; these are combined into the AIC: AIC $=2 k+\chi^{2}$. Each fit is weighted according to

$$
P_{i}=\exp \left[-\left(\mathrm{AIC}_{i}-\min \mathrm{AIC}\right) / 2\right],
$$

where $i$ indexes over all fits being considered. The weights are normalized by the sum over the weights of all fits considered: $w_{i}=P_{i} /\left(\sum_{j} P_{j}\right)$. The blue line and band in Figs. 2 and 3 show the mean and uncertainty of the AICweighted extrapolation. Note that in each panel, we have set the other two parameters to their physical values (e.g., in the leftmost plot, the line and bands are obtained by setting $a=0$ and $L \rightarrow \infty$ ); thus, the line and band are not necessarily expected to pass through the data points, which are at nonzero $a$ and finite $L$. When they do, it shows there is little dependence on the other two parameters. The fitted coefficients also show $c_{a}$ and $c_{V}$ are all consistent with zero within 1 standard deviation. This suggests these ratios have insignificant partial-quenching effects, which are proportional to $O\left(a^{2}\right)$, and there are no detectable finite-volume systematics for the box sizes used. The extrapolated AICweighted values of the ratios are $g_{\Sigma \Sigma} / g_{A}=0.351(6)$ and $g_{\Xi \Xi} / g_{A}=-0.213(5)$, where the systematics from extrapolation have been folded into the statistical uncertainty by the AIC weighting.

We then estimate the contribution of other possible sources of systematic uncertainty: varying the fit ranges of the axial coupling extraction (by increasing and decreasing the two-point fit range by one time step), varying $M_{\pi}^{\mathrm{val}}$ with $M_{\pi}^{\text {sea }}$ (e.g., for a12m310, $M_{\pi}^{\text {sea }}=305.3(4) \mathrm{MeV}$ and $\left.M_{\pi}^{\text {sea }}=310.2 \mathrm{MeV}\right)$, and using different determination of the lattice spacing (using lattice spacing from the physical pion mass ensemble, $0.0871 \mathrm{fm}$, for all a09 ensembles, instead of $0.0888 \mathrm{fm}$ for $\mathrm{a} 09 \mathrm{~m} 310$ and $0.0872 \mathrm{fm}$ for a09m220). This methodological systematic uncertainty is then estimated by taking the difference between the total error from the statistical-only result (difference in quadrature); this gives $g_{\Sigma \Sigma} / g_{A}=0.351(6)(2)$ and $g_{\Xi \Xi} / g_{A}=$ $-0.213(5)(1)$, where the first error includes the statistical and extrapolation uncertainties, and the second error corresponds to systematics associated with varying the lattice parameters. Our results are consistent with the first hyperon axial coupling calculation [19]. In lattice calculations of hyperon axial coupling to date [19-21], only linear extrapolation in $M_{\pi}^{2}$ has been used; lattice spacing $a$ and volume dependence have never been included in the extrapolation. This is also the first work to directly calculate the hyperon charges at the physical pion mass. Compared with these calculations, we achieve significant improvement in statistical uncertainty and control of systematics.

Assuming SU(3) symmetry where $g_{\Sigma \Sigma}=F$ and $g_{\Xi \Xi}=$ $F-D$, we obtain low-energy chiral parameters $D / g_{A}=$ $0.560(9)(2)$ and $F / g_{A}=0.348(7)(1)$, which are not consistent with the determination $D$ but agree with $F$ from semileptonic decay data [2] and PDF values of experimental 


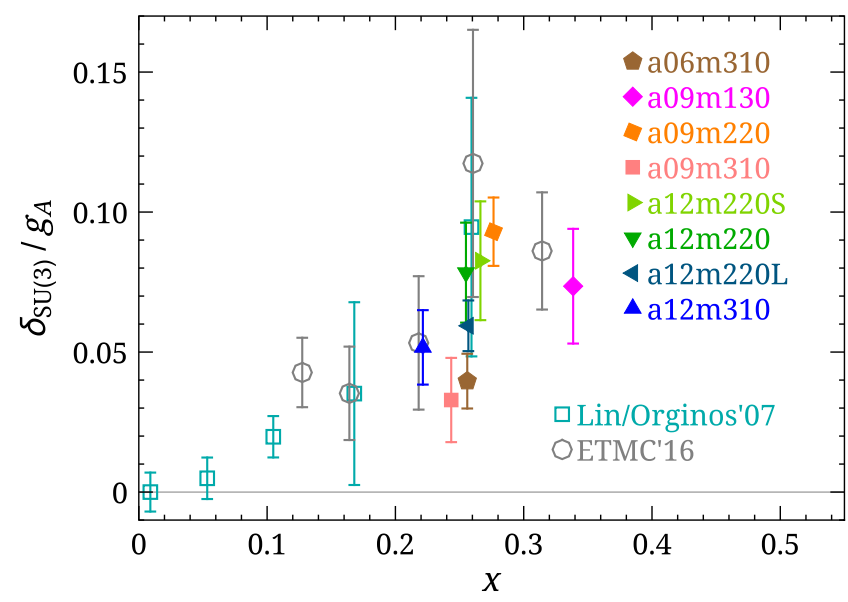

FIG. 4. The ratios of $\mathrm{SU}(3)$ symmetry breaking $\delta_{\mathrm{SU}(3)}$ to the nucleon axial charge $g_{A}$ as a function of $x=\left(M_{K}^{2}-M_{\pi}^{2}\right) /$ $\left(4 \pi^{2} f_{\pi}^{2}\right)$. There is a noticeable increase with $x$ at heavier quark mass, but it is mild when only looking at the lightest two pion masses. We compare our results with the work done by "Lin/ Orginos'07' [19] and ETMC'16 [21].

averaged $g_{A}$ from the Particle Data Guide [31]. In addition, using low-energy constants, we can determine $a_{8}=$ $3 F-D$, which gives the proton spin structure of $\Delta u+\Delta d-$ $2 \Delta s$; our $a_{8} / g_{A}=0.481(15)$ value is consistent with the one commonly used in polarized global fits as a constraint [7].

We also investigate the extent of SU(3) symmetry breaking by considering the quantity

$$
\delta_{\mathrm{SU}(3)}=g_{A}-2 g_{\Sigma \Sigma}+g_{\Xi \Xi} \text {. }
$$

Figure 4 shows $\delta_{\mathrm{SU}(3)} / g_{A}$ for all the ensembles used in this work as a function of the dimensionless quantity $x=$ $\left(M_{K}^{2}-M_{\pi}^{2}\right) /\left(4 \pi^{2} f_{\pi}^{2}\right)$ measured independently on each ensemble. We also shown the same quantities calculated by previous works [19,21], taking the nucleon axial coupling from Table 1 in Ref. [19] and averaged values of 1.15 shown in the work Ref. [21], respectively. We see that these older calculations, mostly with a single lattice spacing, are consistent with the heavier pion masses in our work. Using only the heavier pion masses calculated in the previous studies [19-21], one would draw a conclusion that $\delta_{\mathrm{SU}(3)}$ increases proportional to $x^{2}$. Such a trend perhaps apparent when we look at the heaviest two pion masses in our work. However, the support for such a claim disappears for pion masses between $220 \mathrm{MeV}$ and the physical pion mass. Working directly at the physical pion mass is important to constraining the chiral extrapolation of this symmetry breaking.

Using the $g_{\Sigma \Sigma} / g_{A}$ and $g_{\Xi \Xi} / g_{A}$ found earlier, we obtain $\delta_{\mathrm{SU}(3)} / g_{A}=+0.085(12)$. Thus, we estimate a total SU(3) symmetry breaking size of about $9 \%$, which is smaller than the estimate from Ref. [19].

\section{SUMMARY AND OUTLOOK}

In this work, we have calculated the axial coupling of the $\Sigma$ and $\Xi$ octet baryons using $N_{f}=2+1+1$ lattice QCD. For the first time, not only have these quantities been studied directly at the physical pion mass but also with careful study of the sources of systematic uncertainty, including the lattice-spacing and the finite-volume effects. We calculated multiple source-sink separations for the three-point correlators and used a two-state strategy to fit all separation data simultaneously to remove excited-state contamination. We constructed the ratios of $g_{\Sigma \Sigma} / g_{A}$ and $g_{\Xi \Xi} / g_{A}$ and found these ratios have smaller dependence on the $M_{\pi}^{2}$, lattice spacing $a$, and volume. We then extrapolated the ratios to the physical limit using 12 different fitting forms and combined them using the AIC to obtain $g_{\Sigma \Sigma} / g_{A}=0.351(6)(2)$ and $g_{\Xi \Xi} / g_{A}=-0.213(5)(1)$. We also examined the SU(3) symmetry breaking using these couplings and found around $9 \%$ effect in this updated study, which is about a factor of 2 smaller than the previous lattice study without using physical pion mass.

\section{ACKNOWLEDGMENTS}

We thank the MILC Collaboration for sharing the lattices used to perform this study; the LQCD calculations were performed using the Chroma software suite [32]. This work is supported by Michigan State University through computational resources provided by the Institute for CyberEnabled Research. H. L. is supported by the U.S. National Science Foundation under Grant No. PHY 1653405 "CAREER: Constraining Parton Distribution Functions for New-Physics Searches."
[1] M. J. Savage and J. Walden, Phys. Rev. D 55, 5376 (1997).

[2] N. Cabibbo, E. C. Swallow, and R. Winston, Annu. Rev. Nucl. Part. Sci. 53, 39 (2003).

[3] S. R. Beane, P. F. Bedaque, A. Parreno, and M. J. Savage, Nucl. Phys. A747, 55 (2005).
[4] J. M. Lattimer and M. Prakash, Phys. Rep. 442, 109 (2007).

[5] S. Weissenborn, D. Chatterjee, and J. Schaffner-Bielich, Phys. Rev. C 85, 065802 (2012); 90, 019904(E) (2014).

[6] J. Dai, R. F. Dashen, E. E. Jenkins, and A. V. Manohar, Phys. Rev. D 53, 273 (1996). 
[7] H.-W. Lin et al., Prog. Part. Nucl. Phys. 100, 107 (2018).

[8] R. Gupta, Y.-C. Jang, B. Yoon, H.-W. Lin, V. Cirigliano, and T. Bhattacharya, Phys. Rev. D 98, 034503 (2018).

[9] H.-W. Lin, J.-W. Chen, X. Ji, L. Jin, R. Li, Y.-S. Liu, Y.-B. Yang, J.-H. Zhang, and Y. Zhao, Phys. Rev. Lett. 121, 242003 (2018).

[10] T. Bhattacharya, V. Cirigliano, S. Cohen, R. Gupta, H.-W. Lin, and B. Yoon, Phys. Rev. D 94, 054508 (2016).

[11] T. Bhattacharya, V. Cirigliano, S. Cohen, R. Gupta, A. Joseph, H.-W. Lin, and B. Yoon (PNDME Collaboration), Phys. Rev. D 92, 094511 (2015).

[12] J. R. Green, J. W. Negele, A. V. Pochinsky, S. N. Syritsyn, M. Engelhardt, and S. Krieg, Phys. Rev. D 86, 114509 (2012).

[13] Y. Aoki, T. Blum, H.-W. Lin, S. Ohta, S. Sasaki, R. Tweedie, J. Zanotti, and T. Yamazaki, Phys. Rev. D 82, 014501 (2010).

[14] A. Abdel-Rehim et al., Phys. Rev. D 92, 114513 (2015); 93, 039904(E) (2016).

[15] G. S. Bali, S. Collins, B. Glssle, M. Gckeler, J. Najjar, R. H. Rdl, A. Schfer, R. W. Schiel, W. Sldner, and A. Sternbeck, Phys. Rev. D 91, 054501 (2015).

[16] T. Yamazaki, Y. Aoki, T. Blum, H. W. Lin, M. F. Lin, S. Ohta, S. Sasaki, R. J. Tweedie, and J. M. Zanotti (RBC + UKQCD Collaboration), Phys. Rev. Lett. 100, 171602 (2008).

[17] H.-W. Lin, W. Melnitchouk, A. Prokudin, N. Sato, and H. Shows, Phys. Rev. Lett. 120, 152502 (2018).

[18] S. Aoki et al. (Flavour Lattice Averaging Group), arXiv: 1902.08191 .
[19] H.-W. Lin and K. Orginos, Phys. Rev. D 79, 034507 (2009).

[20] G. Erkol, M. Oka, and T. T. Takahashi, Phys. Lett. B 686, 36 (2010).

[21] C. Alexandrou, K. Hadjiyiannakou, and C. Kallidonis, Phys. Rev. D 94, 034502 (2016).

[22] A. Hasenfratz and F. Knechtli, Phys. Rev. D 64, 034504 (2001).

[23] E. Follana, Q. Mason, C. Davies, K. Hornbostel, G. P. Lepage, J. Shigemitsu, H. Trottier, and K. Wong (HPQCD and UKQCD Collaborations), Phys. Rev. D 75, 054502 (2007).

[24] A. Bazavov et al. (MILC Collaboration), Phys. Rev. D 87, 054505 (2013).

[25] J.-W. Chen, D. O'Connell, and A. Walker-Loud, J. High Energy Phys. 04 (2009) 090.

[26] F.-J. Jiang, arXiv:hep-lat/0703012.

[27] B. Yoon et al., Phys. Rev. D 93, 114506 (2016).

[28] C. Egerer, D. Richards, and F. Winter, Phys. Rev. D 99, 034506 (2019).

[29] J. Green, in 36th International Symposium on Lattice Field Theory (Lattice 2018) East Lansing, MI, United States (Proceedings of Science, 2018).

[30] C. C. Chang et al., Nature (London) 558, 91 (2018).

[31] M. Tanabashi et al. (Particle Data Group), Phys. Rev. D 98, 030001 (2018).

[32] R. G. Edwards and B. Joo (SciDAC, LHPC, and UKQCD Collaborations), Nucl. Phys. B, Proc. Suppl. 140, 832 (2005). 